\title{
Dissipative solitons that cannot be trapped
}

\author{
Rosa Pardo* \\ Departamento de Matemática Aplicada, Facultad de Ciencias Químicas, \\ Universidad Complutense, Avda. Complutense s/n, 28040 Madrid, Spain \\ Víctor M. Pérez-García ${ }^{\dagger}$ \\ Departamento de Matemáticas, Escuela Técnica Superior de Ingenieros Industriales, \\ Universidad de Castilla-La Mancha 13071 Ciudad Real, Spain.
}

( $\Omega$ Dated: November 14, 2006)

\begin{abstract}
We show that dissipative solitons in systems with high-order nonlinear dissipation cannot survive in the presence of trapping potentials of the rigid wall or asymtotically increasing type. Solitons in such systems can survive in the presence of a weak potential but only with energies out of the interval of existence of linear Quantum Mechanical stationary states.
\end{abstract}

PACS numbers: 05.45.Yv, 03.75.Lm, 42.65.Tg

Introduction.- Solitons are self-localized nonlinear waves which are sustained by an equilibrium between dispersion and nonlinearity and appear in a great variety of physical contexts [1]. Solitonlike localized states also arise in dissipative systems driven far from thermal equilibrium such as hydrodynamics [2], granular media [3], gas discharges [4], nonlinear optics and other fields [5-14]. These structures are referred to as "dissipative solitons" and are sustained because of an interplay between dispersion, nonlinearity, gain and losses.

A new family of multidimensional dissipative (bright) solitons has been recently observed and studied in the context of the propagation of optical beams in media with multiphoton absorption [16-19]. These solitons have slowly decaying tails and have a nontrivial flux of energy from their tails to the soliton center where energy is absorbed leading to a stable nonlinear object. In some sense they represent a nontrivial extension of the well-known non-diffracting optical beams [20].

These dissipative soliton-like structures have been also studied in different contexts such as three-dimensional scenarios [21, 22] and should also exist in other physical scenarios ruled by analogous physical phenomena (and model equations) [23].

In this letter we will describe a highly counterintuitive property of these solitons which is that they cannot be trapped by the action of an attractive potential, an affirmation which we will make more precise later. This fact is very striking since attractive potentials are widely used to enhance the stability of conventional nondissipative solitons. In fact, it is well known from Quantum Mechanics that attractive potentials alone without any help of nonlinear interactions are able to support stationary solutions. Even unstable nonlinear structures such as the Townes soliton [23] are stabilized by an external confining potential $[24,25]$.

Mathematical model. - As a mathematical model of solitons under high order dissipation we will use the nonlin- ear Schrödinger equation (NLS)

$$
i \frac{\partial \psi}{\partial t}=-\frac{1}{2} \Delta \psi+V \psi+g|\psi|^{2} \psi+i \gamma|\psi|^{4} \psi
$$

on $\mathbb{R}^{2}$, where $g$ and $\gamma<0$ are real parameters (nonlinearity and dissipation coefficients) and $V$ is a real function describing an external potential acting on the system. Eq. (1) models the propagation of optical beams in media with multiphoton absorption $[16,17,21]$, the mean field dynamics of Bose-Einstein condensates with losses associated to inelastic three-body collisions [22, 26-32] and other physical phenomena [23]. The mathematical studies on this equation have focused on the study of the concentration mechanisms leading to the so called "superstrong" collapse phenomenon (which however, does not involve the formation of a singularity in the mathematical sense) [23, 33, 34].

Stationary solutions.- Stationary solutions of Eq. (1) with $\gamma=0$, and $V=0$ have been discussed extensively since they correspond to the simplest model of nonlinear optical beams and classical superfluids. When $\gamma \neq 0$ they have been studied in Refs. [17, 21, 22]. Let us write

$$
\psi(\boldsymbol{r}, t)=A(\boldsymbol{r}, t) \exp [i \phi(\boldsymbol{r}, t)],
$$

with $A(\boldsymbol{r}, t)>0$. Then Eqs. (1) become

$$
\begin{aligned}
\frac{\partial\left(A^{2}\right)}{\partial t} & =-\nabla \cdot\left(A^{2} \nabla \phi\right)+2 \gamma A^{6}, \\
\frac{\partial \phi}{\partial t} & =\frac{1}{2}\left[\frac{1}{A} \Delta A-(\nabla \phi)^{2}\right]-g A^{2}-V .
\end{aligned}
$$

Stationary solutions of Eq. (1) satisfy $\partial_{t} A=0$, and $\phi(\boldsymbol{r}, t)=\varphi(\boldsymbol{r})-\lambda t$.

It is obvious that $N \equiv\|\psi\|_{2}^{2}=\int_{\mathbb{R}^{2}}|\psi|^{2}=\int_{\mathbb{R}^{2}} A^{2}$, cannot change over stationary solutions. However, let us assume that $N$ is finite for stationary solutions, then using Eq. (1) we get

$$
\frac{d N}{d t}=2 \gamma \int_{\mathbb{R}^{2}} A^{6}>0,
$$


which is a contradiction. This implies that $N$ cannot be finite for nontrivial stationary solutions of Eq. (1). This fact is also known to be characteristic of the linear non-diffracting optical beams [20].

The existence of a nontrivial phase structure $\varphi(\boldsymbol{r})$ is a feature of the case $\gamma \neq 0$ which is not present in the conservative NLS. From Eqs. (3a) and (3b)

$$
\begin{aligned}
\nabla \cdot\left(A^{2} \nabla \varphi\right) & =2 \gamma A^{6}, \\
-\frac{1}{2} \Delta A+\frac{1}{2}(\nabla \varphi)^{2} A+V A & =\lambda A-g A^{3} .
\end{aligned}
$$

In this paper, in order to deal with physically meaningful solutions, we will study solutions of Eqs. (5) which decay asymptotically for large values of $r=\|\boldsymbol{r}\|$, i.e. $A(r) \rightarrow 0$ when $r \rightarrow \infty$.

When the amplitude and phase are radially symmetric non-singular functions $A(\boldsymbol{r})=R(r), \varphi(\boldsymbol{r})=\Phi(r)$, $R^{\prime}(0)=\Phi^{\prime}(0)=0$. Eq. (5a) can be integrated and we get

$$
\Phi^{\prime}(r)=\frac{2 \gamma}{R^{2} r} \int_{0}^{r} \rho R(\rho)^{6} d \rho .
$$

Introducing (6) into (5b) we can write

$$
\begin{aligned}
& -R^{\prime \prime}-\frac{1}{r} R^{\prime}+2 V R= \\
& \quad 2 \lambda R-2 g R^{3}-\frac{4 \gamma^{2}}{R^{3} r^{2}}\left(\int_{0}^{r} \rho R(\rho)^{6} d \rho\right)^{2},
\end{aligned}
$$

complemented with the boundary conditions $R(r) \rightarrow 0$ as $r \rightarrow \infty$ and $R^{\prime}(0)=0$. Eq. (7) is a nonlinear eigenvalue problem with a nonlocal term.

Let us look for solutions satisfying that

$$
Q=2 \gamma \int_{0}^{\infty} \rho R(\rho)^{6} d \rho=\frac{\gamma}{\pi}\|R\|_{L^{6}\left(\mathbb{R}^{2}\right)}^{6},
$$

is finite (mathematically, this means that $A \in L^{6}\left(\mathbb{R}^{2}\right)$ ). In this case Eq. (6) allows us to obtain $\Phi^{\prime}(r) \approx$ $Q /\left[r R^{2}(r)\right]$, for $r \gg 1$, and the asymptotic equation for the amplitude becomes

$$
-R^{\prime \prime}-\frac{1}{r} R^{\prime}+2 V R \simeq 2 \lambda R-2 g R^{3}-\frac{Q^{2}}{R^{3} r^{2}}, \quad r \gg 1 .
$$

System without external potentials $(V=0)$.- When $\lambda>$ 0 and $V=0$ it was shown in Ref. [17] how for large $r$ values the leading order approximation to the solution is $R(r) \sim 1 / \sqrt{r}$. When $\lambda<0$ there are no stationary solutions (this was conjectured in Ref. [17]) and will be proven here for later convenience. First we write $A(\boldsymbol{r}) e^{i \varphi(\boldsymbol{r})}=u+i v$, then Eqs. (1) become

$$
\begin{aligned}
-\frac{1}{2} \Delta u & =\lambda u-g\left(u^{2}+v^{2}\right) u+\gamma\left(u^{2}+v^{2}\right)^{2} v, \\
-\frac{1}{2} \Delta v & =\lambda v-g\left(u^{2}+v^{2}\right) v-\gamma\left(u^{2}+v^{2}\right)^{2} u .
\end{aligned}
$$

in $\mathbb{R}^{2}$, and $u, v \rightarrow 0$ as $|\boldsymbol{r}| \rightarrow \infty$. Let us now consider Eqs. (10) on a generic ball $B_{\rho}$ and let $u, v$ be radially symmetric solutions. Multiplying the first of these equations by $u$, the second by $v$, integrating in $B_{\rho}$ and applying Green formulae we get

$$
\begin{aligned}
\frac{1}{2} \int_{B_{\rho}}|\nabla u|^{2} & +|\nabla v|^{2}-\left.\pi \rho\left(u u_{r}+v v_{r}\right)\right|_{r=\rho} \\
& =\lambda \int_{B_{\rho}} u^{2}+v^{2}-g \int_{B_{\rho}}\left(u^{2}+v^{2}\right)^{2} .
\end{aligned}
$$

Since $R \geq 0$ and $R \rightarrow 0$ at infinity, we can always take a sequence $\rho_{k} \rightarrow \infty$ such that $R_{r}\left(\rho_{k}\right) \leq 0$. However $\left(R^{2}\right)_{r}=2\left(u u_{r}+v v_{r}\right)$, therefore the l.h.s. of Eq. (11) is positive for any $B_{\rho_{k}}$. As a conclusion, if $g \geq 0$ there are no radially symmetric solutions.

When $\lambda<0, g<0$ and $V=0$ there are not radially symmetric solutions with amplitude in $L^{6}\left(\mathbb{R}^{2}\right)$. By comparison results on the Eq. (9), it is not difficult to prove that, when $r \gg 1, R(r) \geq C \exp (\sqrt{-\lambda} r)$ for some $C \in \mathbb{R}$, which is unbounded when $r \rightarrow \infty$ provided $\lambda<0$.

In summary, when $\lambda<0$ there are neither radially symmetric solutions at all for $g \geq 0$ nor solutions satisfying the finiteness requirement for $Q$ (mathematically $A \in L^{6}\left(\mathbb{R}^{2}\right)$ norm) for $g<0$. Thus, we can ensure that there are no physically relevant stationary solutions when $\lambda<0$.

Nonexistence of dissipative solitons in systems with rigid walls.- Now we move to the main point of this paper, which is showing that dissipative solitons of Eq. (1) cannot be trapped by strongly confining potentials, or equivalently proving that stationary solutions of Eq. (3) do not exist for those potentials.

To do so we first consider the case of an infinite potential barrier placed on the boundaries of a finite region $\Omega$. Energy conservation then imposes the condition that $u(\boldsymbol{r})=0$ for $\boldsymbol{r} \in \partial \Omega$.

We start from Eqs. (10), multiply Eq. (10a) by $v$, Eq. (10b) by $u$, and integrate over $\Omega$ to get

$$
\begin{aligned}
\frac{1}{2} \int_{\Omega} \nabla u \cdot \nabla v= & \lambda \int_{\Omega} u v-g \int_{\Omega}\left(u^{2}+v^{2}\right) u v \\
& +\gamma \int_{\Omega}\left(u^{2}+v^{2}\right)^{2} v^{2} \\
\frac{1}{2} \int_{\Omega} \nabla u \cdot \nabla v= & \lambda \int_{\Omega} u v-g \int_{\Omega}\left(u^{2}+v^{2}\right) u v \\
& -\gamma \int_{\Omega}\left(u^{2}+v^{2}\right)^{2} u^{2}
\end{aligned}
$$

The difference of Eqs. (12a) and (12b) leads to

$$
\gamma \int_{\Omega}\left(u^{2}+v^{2}\right)^{3}=0
$$

from where we conclude that either $\gamma=0$ or the only solution is the trivial one $u=v=0$. Thus dissipative solitons cannot exist when confined to a finite region. 
We can provide some extra results valid for more general geometries of the domain $\Omega$. Let us consider again Eqs. (10). Multiplying Eq. (10a) by $u$, Eq. (10b) by $v$, integrating in $\Omega$ and applying Green formulae we get

$\frac{1}{2} \int_{\Omega}|\nabla u|^{2}=\lambda \int_{\Omega} u^{2}-g \int_{\Omega}\left(u^{2}+v^{2}\right) u^{2}+\gamma \int_{\Omega}\left(u^{2}+v^{2}\right)^{2} u v$,

$\frac{1}{2} \int_{\Omega}|\nabla v|^{2}=\lambda \int_{\Omega} v^{2}-g \int_{\Omega}\left(u^{2}+v^{2}\right) v^{2}-\gamma \int_{\Omega}\left(u^{2}+v^{2}\right)^{2} u v$.

Adding both equations we obtain

$$
\frac{1}{2} \int_{\Omega}|\nabla u|^{2}+|\nabla v|^{2}=\lambda \int_{\Omega} u^{2}+v^{2}-g \int_{\Omega}\left(u^{2}+v^{2}\right)^{2} .
$$

Poincare's inequality on bounded domains $\Omega$ says that if $\delta_{1}$ is the first eigenvalue of the Laplacian operator on $\Omega$ with Dirichlet homogeneous boundary conditions, then

$$
\delta_{1}=\inf _{\phi \in H_{0}^{1}(\Omega)} \frac{\int_{\Omega}|\nabla \phi|^{2}}{\int_{\Omega}|\phi|^{2}} .
$$

This inequality also holds for unbounded domains $\Omega$, which are bounded along one of the directions, which allows us to extend our results to systems with strong confinement along a single direction. In any of those situations we will have

$$
\begin{aligned}
\frac{\delta_{1}}{2} \int_{\Omega}\left(u^{2}+v^{2}\right) & \leq \frac{1}{2} \int_{\Omega}|\nabla u|^{2}+|\nabla v|^{2} \\
& =\lambda \int_{\Omega}\left(u^{2}+v^{2}\right)-g \int_{\Omega}\left(u^{2}+v^{2}\right)^{2},
\end{aligned}
$$

from where we find that

$$
\left(\frac{\delta_{1}}{2}-\lambda\right) \int_{\Omega}\left(u^{2}+v^{2}\right) \leq-g \int_{\Omega}\left(u^{2}+v^{2}\right)^{2},
$$

so that we get that when $g>0$ the negativity of the second term implies that $\lambda>\delta_{1} / 2$, thus if $\lambda<\delta_{1} / 2$ the only solution is $u=v=0$. Moreover $\delta_{1}(\Omega) \rightarrow 0$ when $|\Omega| \rightarrow \infty$, which matches our previous results on radially symmetric stationary solutions. However, here the result is proven rigorously for any type of solution (not only symmetric ones) in hard-wall type potentials corresponding to semi-infinite regions.

Systems with nonsingular confinement .- We have already shown that dissipative solitons of Eq. (1) cannot be confined to finite spatial regions by hard-wall type potentials. In what follows we will study the effect of non-singular potentials. To do so we will first start by considering unbounded asymptotically increasing potentials i.e. potentials with $V \rightarrow \infty$ as $r \rightarrow \infty$, such as those of the form $0<V(r) \sim r^{p}$ with $p>0$. This is the case of applications to Bose-Einstein condensates in magnetic traps, where usually $V(r) \sim r^{2}$. These potentials provide a strong confinement and in the linear $(g=0, \gamma=0)$ case have an infinite number of bound states.
Let us then consider the version of Eq. (11) including the potential term. The same type of arguments used for the case $V=0, \lambda<0$ can be used to prove that if $g \geq 0$

$$
\lambda \geq \frac{\int_{B_{\rho_{k}}} V R^{2}}{\int_{B_{\rho_{k}}} R^{2}} .
$$

As stated before, stationary solutions must have necessarily $\int_{B_{\rho}} R^{2} \rightarrow \infty$ when $\rho \rightarrow \infty$. For asymptotically increasing potentials there is some $r=r_{*}$ such that for all $r>r_{*}, V\left(r_{*}\right)>\lambda$ and then both the numerator and denominator of r.h.s. of (18) diverge and using L'Hopital rule

$$
\lim _{\rho \rightarrow \infty} \frac{\int_{B_{\rho}} V R^{2}}{\int_{B_{\rho}} R^{2}}=\lim _{\rho \rightarrow \infty} \frac{V R^{2}}{R^{2}},
$$

therefore $\lambda \geq \lim _{\rho \rightarrow \infty} V(\rho)=\infty$ and as a conclusion, there are no radially symmetric solutions.

When $g<0$, there are not radially symmetric solutions with amplitude in $L^{6}\left(\mathbb{R}^{2}\right)$. The reason is again that, when $r \gg 1, R(r) \geq C \exp (\sqrt{-\lambda} r)$ for some constant $C$, which is unbounded when $r \rightarrow \infty$. This excludes the possibility of existence of physically meaningful stationary solutions for both signs of the nonlinear coefficient $g$ and implies that dissipative solitons of Eq. (1) cannot exist when an asymptotically increasing potential is present.

The case more relevant for physical applications is that of bounded potentials, i.e. $V(r) \rightarrow V_{\infty}$ when $r \rightarrow \infty$. We can argue as in the previous case that $\lambda \geq \lim _{\rho \rightarrow \infty} V=V_{\infty}$, thus implying the possibility of existence of solitary waves only for values of the eigenvalue $\lambda$ satisfying $\lambda \geq V_{\infty}$. In that situation the asymptotic analysis of the solutions for $r \gg 1$ is the same presented in Refs. $[17,22]$ and we can expect the existence of solutions which will be close to those of the case $V=0$ but with local deformations in the region where $V$ has appreciable values.

This is a very striking result since when $\gamma=0$ stationary localized solutions can exist only when $\lambda \leq V_{\infty}$. In the language of Quantum Mechanics bound states lie in the region of energies $E \leq V_{\infty}$ and for values of the eigenvalue $E>V_{\infty}$ only delocalized plane-wave type states exist. Our result is exactly the opposite: no matter what small is $\gamma$ localized solutions can exist only when $E \equiv \lambda>V_{\infty}$, so the "energies" of localized solutions (solitons) in this nonlinear model with dissipation are necessarily above the limit for existence of solutions in the linear case.

Physical implications and soliton mobility.- These results have practical implications for the experimental generation of dissipative solitons in Bose-Einstein condensates with appreciable losses due to three-body collisions. In realistic condensates, due to the condition $N<\infty$ we can only construct dissipative structures close to the stationary solutions but with a finite lifetime 
[22]. In fact, numerical simulations of blow up phenomena with high order losses show the formation of multiring structures whose shape resemble that of dissipative solitons $[22,27]$. Our results imply that the magnetic confining potentials of the form $V(r) \sim r^{2}$ are not appropriate to confine the atomic cloud if these dissipative solitons are to be obtained. In this sense it would be more convenient to use a laser beam to generate an optical dipole type trap, of the form $V(r) \sim e^{-r^{2}}$ thus being compatible with the existence of stationary structures.

Could a localized potential be used to control these solitons? The problem of soliton mobility is an interesting one in many different contexts [35]. In our case an optical dipole trap (or any other "localized potential") cannot probably be used to move the soliton. The reason is that these dissipative solitons arise as a global equilibrium between an inward flux of particles due to the nontrivial phase structure and the central peak at which particles are dissipated [22]. So, even if a local potentials could be used to move the central peak, the refilling mechanism would lead to a new soliton at the center [18] while the moving part would experience a fast decay due to losses. The only possibility for moving this soliton would be to use a bounded potential (to ensure existence) having a very slow decay ensuring a global, although small, action on the dissipative soliton (i.e. on the same scale of the soliton). An example could be a potential of Yukawa type $V(r) \sim[1-\exp (-r)] / r$.

Conclusions.- We have studied a class dissipative solitons in models which have received a lot of attention in the last years in different physical contexts $[16,17,21-$ 23, 26-34]. Contrary to the intuition and the observed typical behavior of solitons these dissipative solitons cannot be trapped by using strongly confining potentials. This fact represents a notable exception to the typical behavior of solitons in the context of systems ruled by nonlinear Schrödinger equations. Moreover in the case of weakly confining potentials the solitons can exist only in the region of eigenvalues (energies) without bound states in the linear regime. We have also discussed some physical implications of our findings and made some conjectures on the mobility of these solitons on the basis of our findings.

This work has been partially supported by grants BFM2003-02832 and MTM2006-08262 (Ministerio de Educación y Ciencia, Spain) and PAI-05-001 (Junta de Comunidades de Castilla-La Mancha).

* Electronic address: rpardo@mat.ucm.es

$\dagger$ Electronic address: victor.perezgarcia@uclm.es

[1] A. Scott, Nonlinear Science: Emergence and dynamics of coherent structures, Oxford Appl. and Eng. Mathematics, Vol. 1, Oxford (1999).
[2] O. Lioubashevski, H. Arbell, and J. Fineberg, Phys. Rev. Lett. 76, 3959 (1996).

[3] P. B. Umbanhowar, F. Melo, and H. L. Swinney, Nature (London) 382, 793 (1996).

[4] C. P. Schenk et al., Phys. Rev. E 57, 6480 (1998).

[5] N. N. Rosanov and G. V. Khodova, J. Opt. Soc. Am. B 7, 1057 (1990).

[6] N. N. Akhmediev, V. V. Afanasjev, and J. M. SotoCrespo, Phys. Rev. E 53, 1190 (1996).

[7] W. J. Firth and A. J. Scroggie, Phys. Rev. Lett. 76, 1623 (1996).

[8] S. R. Marder et al., Science 276, 1233 (1997).

[9] J. M. Soto-Crespo, N. Akhmediev, and A. Ankiewicz, Phys. Rev. Lett. 85, 2937 (2000).

[10] S. Barland et al., Nature (London) 419, 699 (2002).

[11] U. Bortolozzo et al., Phys. Rev. Lett. 93, 253901 (2004).

[12] K. Staliunas, Phys. Rev. Lett. 91, 053901 (2003).

[13] S. Saprykin, E. A. Demekhin, and S. Kalliadasis, Phys. Rev. Lett. 94, 224101 (2005).

[14] H. Michinel, M. J. Paz-Alonso, V. M. Pérez-García, Phys. Rev. Lett. 96023903 (2006).

[15] V. Skarka, N. B. Aleksic, Phys. Rev. Lett. 96, 013903 (2006).

[16] A. Dubietis, et al., Phys. Rev. Lett. 92, 253903 (2004).

[17] M. A. Porras, et al., Phys. Rev. Lett. 93, 153902 (2004).

[18] A. Dubietis et al., Opt. Lett. 29, 2893 (2004).

[19] P. Polesana et al., Opt. Express 13, 6160 (2005).

[20] J. Durnin, J. Opt. Soc. Am. A 4651 (1987); P. Johannisson, et al., Opt. Commun. 222, 107 (2003).

[21] M. A. Porras, A. Parola and P. Di Trapani, J. Opt. Soc. Am. B 22, 1406 (2005).

[22] A. Alexandrescu, V. M. Pérez-García, Phys. Rev. A 73, 053610 (2006).

[23] C. Sulem, P. Sulem, The Nonlinear Schrödinger Equation: Self-focusing and Wave Collapse, Springer, Berlin, 2000.

[24] L. Berge, T. J. Alexander, and Yu. S. Kivshar, Phys. Rev. A 62, 023607 (2000).

[25] T. Tsurumi and M. Wadati, J. Phys. Soc. Jpn. 66, 3035 (1997); ibid. 68, 1531 (1999).

[26] H. Saito and M. Ueda, Phys. Rev. Lett. 861406 (2001).

[27] H. Saito and M. Ueda, Phys. Rev. A 65033624 (2002).

[28] S. K. Adhikari, Phys. Rev. A 66, 013611 (2002).

[29] S. Métens, G. Dewel, and P. Borckmans, Phys. Rev. A 68045601 (2003).

[30] L. Salasnich, A. Parola, and L. Reatto, Phys. Rev. Lett. 91, 080405 (2003).

[31] C. M. Savage, N. P. Robins, and J. J. Hope, Phys. Rev. A 67, 014304 (2003).

[32] S. K. Adhikari, J. Phys. B: At. Mol. Opt. Phys. 37 (2004) 1185.

[33] N. E. Kosmatov, V. F. Shvets, and V. E. Zakharov, Physica D (Amsterdam) 5216 (1991).

[34] B. J. LeMesurier, Physica D (Amsterdam) 138 (2000) 334; Math. Comput. Simul. 55 (2001) 503.

[35] See e.g. Z. Xu, Y. V. Kartashov, and Ll. Torner, Phys. Rev. Lett. 95, 113901 (2005); V. A. Brazhnyi, V. V. Konotop, and V. M. Pérez-García, Phys. Rev. Lett. 96, 060403 (2006); M. Matuszewski et al., Phys. Rev. A 73, 063621 (2006); P. Pedri and L. Santos, Phys. Rev. Lett. 95200404 (2005); R. Fischer, et al., Phys. Rev. Lett. 96, 023905 (2006), and references therein. 